\title{
SURFACE TEMPERATURE DETERMINATION FROM BOREHOLE MEASUREMENTS: REGULARIZATION AND ERROR ESTIMATES
}

\author{
TRAN THI LE \\ and \\ MILAGROS NAVARRO \\ HoChiMinh City University \\ Department of Mathematics \\ University of the Philippines \\ Department of Mathematics \\ HoChiMinh City, VIETNAM \\ Diliman, Quezon City, PHILIPPINES
}

(Received July 15. 1993 and in revised form March 30. 1994)

\begin{abstract}
The authors consider the problem of determining the temperature distribution $u(x, t)$ on the half-line $x=0, t>0$, from measurements at an interior point, for all $t>0$. As is well-known, this is an ill-posed problem Using the Tikhonov method, the authors give a regularized solution, and assuming the (unknown) exact solution is in $H^{\alpha}(\mathbb{R}), \alpha>0$ They give an error estimate of the order $1 /(1 n 1 / \varepsilon)^{\alpha}$ for $\varepsilon \rightarrow 0$, where $\varepsilon>0$ is a bound on the measurement error
\end{abstract}

KEY WORDS AND PHRASES. Surface temperature distribution, Tikhonov regularization, error estimate.

1991 AMS SUBJECT CLASSIFICATION CODES. 35R25, 35K05

\section{INTRODUCTION.}

We consider the problem of determining the temperature distribution $u(x, t)$ on the half line $x=0, t>0$ from measurements at $x=1$ for all $t>0$ The problem is of importance in Geophysics. It has been considered by various authors (see references) Of particular relevance to the present work are the papers of Anderssen and Saull [1] and of Anderssen [2], and the paper of Carasso [3]. The paper of Anderssen and the one of Anderssen and Saull. give the physical background and concentrate on numerical and stabilization problems. The work of Carasso makes use of the Tikhonov regularization method. The difference between Carasso's result and ours can best be seen in Remark 2 at the end of this paper. The paper of Talenti and Vessella [4] is also of interest, it gives stability estimates between the two solutions We should note that the problem under consideration is ill-posed in the sense that a solution does not always exist, and that whenever they exist, solutions do not depend continuously on the given (measured) data. The ill-posedness is discussed by various authors (see [1], [3] and [5]). We shall regularize the problem using the Tikhonov method. We shall give error estimates, assuming the exact solution can be extended to a function in $H^{\alpha}(\mathbb{R}), \alpha>0$.

2. MAIN RESULTS.

We propose to solve the heat equation

$$
u_{t}-u_{x x}=0 \quad 0<x<\infty, \quad t>0,
$$

subject to the following conditions

$$
u(x, 0)=f(x), \quad u(1, t)=g(t),
$$


and a decay condition. which. following [5]. we take as

$$
\lim _{x \rightarrow \infty} x^{x^{2}} \ln \left(\int_{0}^{l} a^{2} u^{2}(x, t ; d t)=0 \quad \text { for any } \Gamma>0\right.
$$

If we write $v(t)$ for $u(0, t)$, then we have the following representation formula, alid for $u(x, 0)=0$. which, without loss of generality, we assume here

$$
u(x, t)=\int_{0}^{t} v(s) K(x, t-s) d s
$$

where (see Widder [6] and Cannon [7])

$$
\begin{aligned}
K(x, t) & =(4 \pi)^{-1} x t^{-3 / 2} \exp \left(-x^{2} / 4 t\right) & & \text { if } t>0 \\
& =0 & & \text { if } t \leq 0
\end{aligned}
$$

To find $v(t)$, it is therefore sufficient to solve the following integral equation

$$
(4 \pi)^{-1} \int_{0}^{t} v(s)(t-s)^{-3 / 2} \exp (-1 / 4(t-s))=g(t), \quad t>0
$$

which is conveniently written as

$$
\frac{1}{\sqrt{2 \pi}} \int_{0}^{t} v(s) K(t-s)=h(t) . \quad t>0
$$

where

$$
\begin{aligned}
h(t) & =2 \sqrt{2 \pi} g(t) & & \\
K(t) & =t^{-3 / 2} \exp (-1 / 4 t), & & t>0 \\
& =0, & & t \leq 0
\end{aligned}
$$

We assume $g(t)$ to be an $L_{2}$-function, and, hence, $h(t)$ is an $L_{2}$-function. Taking the Fourier transform of both sides of (2.7) gives

$$
\widehat{K}(p) \widehat{v}(p)=\widehat{h}(p)
$$

where

$$
\widehat{K}(p)=\frac{1}{\sqrt{2 \pi}} \int_{-\infty}^{\infty} K(t) e^{-\imath p t} d t
$$

and similarly for $h$ and $v$, which are understood to vanish for $t<0$. We shall consider the following regularized equation

$$
\beta \widehat{v}_{\beta}+|\widehat{K}|^{2} \widehat{v}_{\beta}=\bar{K} \widehat{h}, \quad \beta>0,
$$

Note that a solution $\widehat{v}_{\beta}$ of (2.10) always exists and is Lipschitzian in $\widehat{h}$ The inverse Fourier transform $v_{\beta}$ of $\widehat{v}_{\beta}$, which we shall take as our regularized solution for an appropriate $\beta$, is therefore Lipschitzian in $h$ since the $L_{2}$-Fourier transform is a unitary operator on $L_{2}(\mathbb{R})$.

Now, let $v$ be the exact solution of (2.7) for $h=h_{0}$, and suppose the error between the measured right hand side of (2.7) and the (unknown) exact value is less than $\varepsilon>0$, i.e.,

$$
\left|h-h_{0}\right|_{2}<\varepsilon \text { where }|\cdot|_{2}=L_{2} \text {-norm }
$$

Then, we have the following error estimate, taking $\beta=\varepsilon$

THEOREM 2.1. Let (2.11) hold, and suppose $v$ is in $H^{\alpha}(\mathbb{R}), \alpha>0$ Then

$$
\left|v_{\varepsilon}-v\right|,<c /(1 n \cdot 1 / \varepsilon)^{\varepsilon \alpha}
$$


where

$$
v_{\varepsilon}(t)=\frac{1}{\sqrt{2 \pi}} \int_{-\alpha}^{x} \widehat{v}_{\varepsilon}(p) \exp (i p t) d p
$$

and $c=2^{1 / 2} B$ with $B$ defined in (2 20)

PROOF. By (2 8) and (29), we have for $\beta>0$

$$
\beta\left(\widehat{v}_{\beta}-\widehat{v}\right)+|\widehat{K}|^{2}\left(\widehat{v}_{\beta}-\widehat{v}\right)=-\beta \widehat{v}+\overline{\widehat{K}}\left(\widehat{h}-\widehat{h}_{0}\right)
$$

Taking the $L_{2}$-inner product with $\hat{v}_{3}-\widehat{v}$ and estimating, we have the inequality

$$
\begin{gathered}
\beta\left|\widehat{v}_{3}-\widehat{v}\right|_{2}^{2}+\left|\widehat{K}\left(\widehat{v}_{3}-\widehat{v}\right)\right|_{2}^{2} \leq \beta|\widehat{v}|_{2}\left|\widehat{v}_{\beta}-\widehat{v}\right|_{2}+\left|\widehat{h}-\widehat{h}_{0}\right|_{2} \mid \widehat{K}\left(\widehat{v}_{\beta}-\left.\left.\widehat{v}\right|_{2}\right|_{2}\right. \\
\leq \beta|\widehat{v}|_{2}^{2}+\frac{1}{2} \beta\left|\widehat{v}_{3}-\widehat{v}\right|_{2}^{2}+\frac{1}{2}\left(\left|\widehat{h}-\widehat{h}_{0}\right|_{2}^{2}+\left|\widehat{K}\left(\widehat{v}_{\beta}-\widehat{v}\right)\right|_{2}^{2}\right)
\end{gathered}
$$

Letting $\beta=\varepsilon<1$, and noting that

$$
\left|\widehat{h}-\widehat{h}_{0}\right|_{2}=\left|h-h_{0}\right|_{2}<\varepsilon
$$

we then have. after some rearrangements

$$
\varepsilon\left|\widehat{v}_{\varepsilon}-\widehat{v}\right|_{2}^{2}+\left|\widehat{K}\left(\widehat{v}_{\varepsilon}-\widehat{v}\right)\right|_{2}^{2} \leq \varepsilon\left(|\widehat{v}|_{2}^{2}+1\right)
$$

In particular,

$$
\varepsilon\left|\widehat{v}_{\bar{\varepsilon}}-\widehat{v}\right|_{2}^{2} \leq \varepsilon\left(|\widehat{v}|_{2}^{2}+1\right)
$$

and

$$
\left|\widehat{K}\left(\widehat{v}_{\varepsilon}-\widehat{v}\right)\right|_{2}^{2} \leq \varepsilon\left(|\widehat{v}|_{2}^{2}+1\right)
$$

Now, taking the $L_{2}$-inner product of both sides of (2.13) with $|p|^{2 \alpha}\left(\widehat{v}_{\varepsilon}-\widehat{v}\right)$ and estimating, we have (for $\beta=\varepsilon$ ) the inequality

$$
\begin{aligned}
& \left.\left.\varepsilon|| p\right|^{\alpha}\left(\widehat{v}_{\varepsilon}-\widehat{v}\right)\right|_{2} ^{2}+\left.\left.|| p\right|^{\alpha} \widehat{K}\left(\widehat{v}_{\varepsilon}-\widehat{v}\right)\right|_{2} ^{2} \\
& \leq\left.\left.\left.\left.\varepsilon|| p\right|^{\alpha} \widehat{v}\right|_{2}|| p\right|^{\alpha}\left(\widehat{v}_{\varepsilon}-\widehat{v}\right)\right|_{2}+\left.\left.\left.\left.|| p\right|^{\alpha} \widehat{K}\right|_{\infty}\left|\widehat{h}-\widehat{h}_{0}\right|_{2}|| p\right|^{\alpha}\left(\widehat{v}_{\varepsilon}-\widehat{v}\right)\right|_{2}
\end{aligned}
$$

In view of the following result (see [8])

$$
|\widehat{K}(p)|=2 \exp \left(-(|p| / 2)^{1 / 2}\right)
$$

we have

From (2.18), we have, in particular

$$
\left.\left.|| p\right|^{\alpha} \widehat{K}\right|_{\infty}<\infty
$$

$$
\left.\left.\varepsilon|| p\right|^{\alpha}\left(\widehat{v}_{\varepsilon}-\widehat{v}\right)\right|_{2} ^{2} \leq\left.\left.\varepsilon|| p\right|^{\alpha}\left(\widehat{v}_{\varepsilon}-\widehat{v}\right)\right|_{2}\left(\left.\left.|| p\right|^{\alpha} \widehat{K}\right|_{\infty}+\left.\left.|| p\right|^{\alpha} \widehat{v}\right|_{2}\right)
$$

i.e.,

$$
\left.\left.|| p\right|^{\alpha}\left(\widehat{v}_{\varepsilon}-\widehat{v}\right)\right|_{2} \leq\left.\left.|| p\right|^{\alpha} \widehat{K}\right|_{\infty}+\|v\|_{\alpha}
$$

where

$$
\left.\left.\|v\|_{\infty} \equiv|| p\right|^{\alpha} \widehat{v}\right|_{2} \quad \text { (the } H^{\alpha} \text {-norm). }
$$

Let

$$
B^{2}=2\left(\left.\left.|| p\right|^{\alpha} \widehat{K}\right|_{\infty} ^{2} 2\|v\|_{\alpha}^{2}\right)+1
$$

Then, we have

$$
\left|v_{\varepsilon}-v\right|_{2}+\left\|v_{\varepsilon}-v\right\|_{\alpha} \leq 2 B
$$

The foregoing step establishes an $H^{\alpha}$-bound on $v_{\varepsilon}-v$. As a final step in our proof, we estimate the smallness of $\left|v_{\varepsilon}-v\right|_{2}$ for $\varepsilon \rightarrow 0$. To this end, we have for any $a>0$

$$
\begin{aligned}
\int_{|p| \leq a}\left|\widehat{v}_{\varepsilon}-\widehat{v}(p)\right|^{2} d p & \leq \int_{|p| \leq a}|\widehat{K}(p)|^{2} /\left.\widehat{K}(a)\right|^{2}\left|\left(\widehat{v}_{\varepsilon}-\widehat{v}\right)(p)\right|^{2} d p \\
& \leq \exp (a / 2)^{1 / 2} B^{2} \varepsilon
\end{aligned}
$$


On the other hand

$$
\int_{\mid p_{i} \geq a}\left|\left(\widehat{v}_{\bar{c}}-\widehat{v}\right)(p)\right|^{2} d p \leq\left.\left. a^{-2 \alpha} \int_{x}^{x}|| p\right|^{\alpha}\left(\widehat{v}_{z}-\widehat{v}\right)\right|^{2} d p \leq B^{2} / a^{2 \alpha}
$$

Now, let $a=p_{\varepsilon}$ be the positive solution of the equation

$$
|p|^{20} \exp (|p| / 2)^{1,2}=1 / \varepsilon
$$

Then

$$
\left|\widehat{v}_{\varepsilon}-\widehat{v}\right|_{2}^{2} \leq 2 B^{2} / p_{\varepsilon}^{2 \alpha}
$$

Taking the logarithm of each side of (2 22) gives

$$
2 \alpha \ln p_{\varepsilon}+\left(p_{\varepsilon} / 2\right)^{1 / 2}=\ln (1 / \varepsilon)
$$

Since $p_{\varepsilon} \rightarrow \infty$ for $\varepsilon \rightarrow 0$, it follows that, for all $\varepsilon>0$ sufficiently small, the left-hand side of (2.24) is less than $p_{\varepsilon}^{1 / 2}$ or equivalently

$$
\left(1 / p_{\varepsilon}^{2}\right)<1 /(\ln 1 / \varepsilon)^{4}
$$

Hence, for all small $\varepsilon>0$, we have from (2.23)

$$
\left|v_{\varepsilon}-v\right|_{2}^{2}=\left|\widehat{v}_{\varepsilon}-\widehat{v}\right|_{2}^{2}=c^{2} j(\ln 1 / \varepsilon)^{4 \alpha}, \quad c^{2}=2 B^{2}
$$

This completes the proof.

REMARK 1. Among all choices of $\beta=\varepsilon^{\delta}, \delta>0$, as Tikhonov parameters, our choice $\beta=\varepsilon$, in a sense, is, from the point of view of our analysis, the optimal one, except for the coefficient $c$ in the inequality (2.12). First, if $\delta>1$, then our analysis breaks down, since the steps leading to (2.19) are no longer valid. If $0<\delta<1$, then, retracing the steps from (2.13) to (2.24), we would have, for $\beta=\varepsilon^{\delta}$, an estimate of the form

$$
\left|v_{\beta}-v\right|_{2} \leq c^{\prime}\left(\|v\|_{\alpha}, \varepsilon\right) /(\delta \ln 1 / \varepsilon)^{2 \alpha}
$$

where $c^{\prime}$ is bounded as $\varepsilon \rightarrow 0$. We omit the details there.

REMARK 2. In [3], Carasso (under weaker conditions on the exact solution) produced a regularized solution that approximates the exact solution only at interior points $x>0$.

In closing, we would like to point out that multidimensional analogues of our problem are much more complicated. They are considered, e.g., in [9], where the problem is treated numerically, and in [10], where the problem is formulated as a moment problem.

ACKNOWLEDGEMENT. The authors would like to thank the referee for the most valuable comments and suggestions for improvement of the paper. The work of the first author was completed with finanical support from the National Basic Research Program in the Natural Sciences of Vietnam. 


\section{REFERENCES}

1 ANDERSSEN, R S and SAULL, V A, Surface temperature history determination from Borehole measurements, Mathematical Geology 5 (1973)

2 ANDERSSEN, R S , A review of numerical methods for certain improperly posed parabolic partial differential equations Australian National University Computer Centre. Technical Report No 36, page 47, 1970

3 CARASSO, A, Determining surface temperatures from interior observations, SIAM J. Appl. Math. 42 (1982), 558-574

4 TALENTI. G, Sui problemı mal posti, Boll. Un Mat. Ital A 15 (1978), 1-29

5 TALENTI, G and VESSELLA. S. Note on an ill-posed problem for the heat equation. $j$. Austral. Math. Soc.. A32 (1982), 358-368

6 WIDDER. D V, The Heat Equation, Academic Press, New York, 1975

7 CANNON, J R, The One Dimensional Heat Equatıon, Addison-Wesley. Menlo Park. 1984.

8 ERDELYI, A, Tables of Integral Transforms, Vol 1, pages 16 and 75. MacGraw-Hill, 1954.

9. CIALKOWSKI, M J., GRYSA, K and JANKOWSKI. J . Inverse problems of the linear nonstationary heat transfer equations, Z. angew. Math. Mech. 72 (1992). T61 1-T614

10 ANG, D D ; LE, T.T and THANH, D N, Multidimensional surface temperature determination (in preparation)

11. CANNON, J R, A Cauchy problem for the heat equation, Ann. Mat. Pura Appl. 66 (1964), 155-166.

12. CANNON, J.R. and KLEIN, R E, Optimal selection of measurements locations in a conductor for approximate determination of temperature distributions, J. Dyn. Syst. Meas. and Control 93 (1971), 193-199

13. TIKHONOV, A.A. and GLASKO, V.V , Methods of determining the surface temperature of a body, Z. Vycisl. Mat. I Mat. Flz. 7 (1967), 910-914

14. HAO, D N and GORENFLO, R, A noncharacteristic Cauchy problem for the heat equation, Acta Appl. Math., 24 (1991), 1-27

15. TIKHONOV, A.N. and ARSENIN, I.V., Solutions of Ill-Posed Problems, Wiley, New York, 1977. 


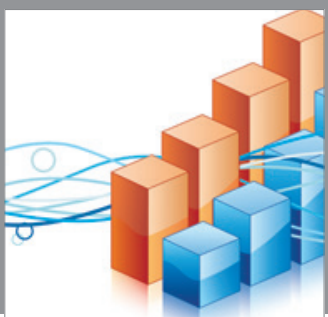

Advances in

Operations Research

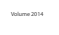

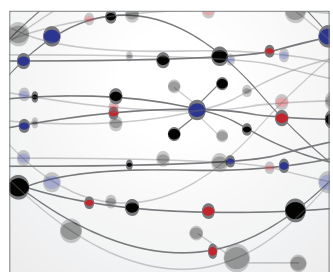

\section{The Scientific} World Journal
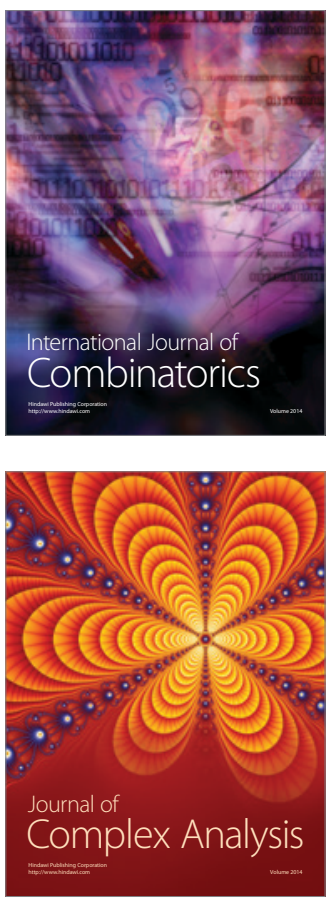

International Journal of

Mathematics and

Mathematical

Sciences
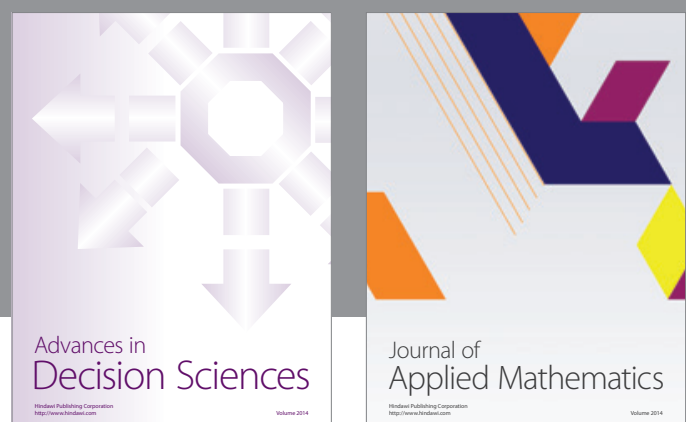

Journal of

Applied Mathematics
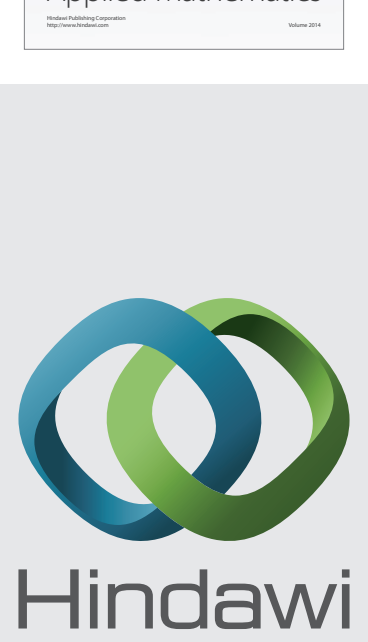

Submit your manuscripts at http://www.hindawi.com
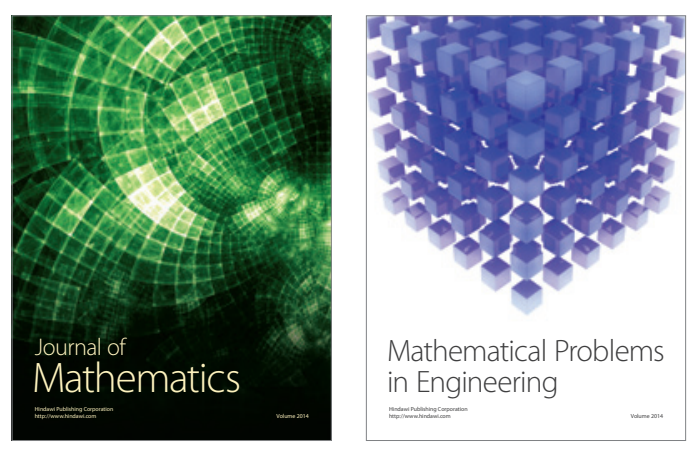

Mathematical Problems in Engineering
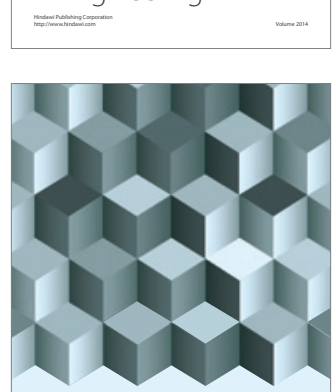

Journal of

Function Spaces
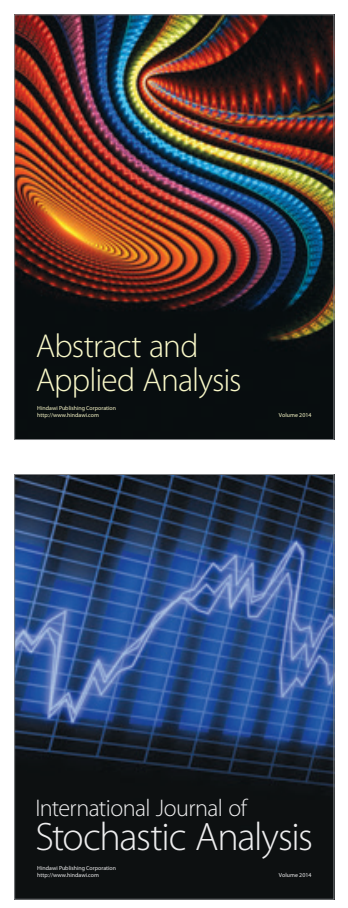

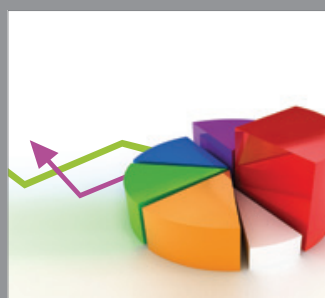

ournal of

Probability and Statistics

Promensencen
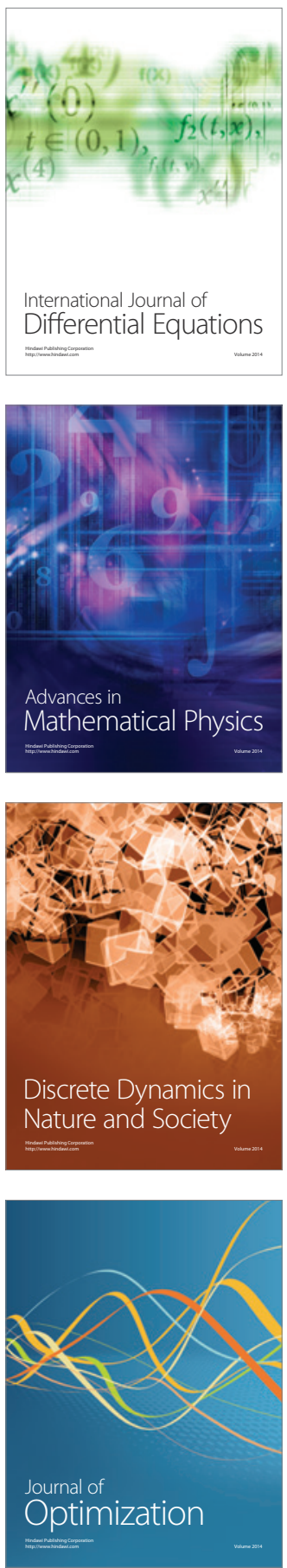